\title{
Characterization of an A-kinase anchoring protein-like suggests an alternative way of PKA anchoring in Plasmodium falciparum
}

\author{
Kossiwa Bandje 1,2,3, Bernina Naissant 2,3,4,5, Pascal Bigey ${ }^{2,6}$, Murielle Lohezic ${ }^{1,2,3}$, Marlène Vayssières ${ }^{2,7}$, \\ Magali Blaud ${ }^{2,7}$, Laetitia Kermasson 1,2,3, José-Juan Lopez-Rubio ${ }^{8}$, Gordon Langsley 2,4,5,9, \\ Catherine Lavazec ${ }^{2,3,4,5,10}$, Philippe Deloron ${ }^{1,2,3}$ and Anaïs Merckx ${ }^{1,2,3^{*}}$
}

\begin{abstract}
Background: The asexual intra-erythrocytic multiplication of the malaria parasite Plasmodium falciparum is regulated by various molecular mechanisms. In eukaryotic cells, protein kinases are known to play key roles in cell cycle regulation and signaling pathways. The activity of CAMP-dependent protein kinase (PKA) depends on A-kinase anchoring proteins (AKAPs) through protein interactions. While several components of the CAMP dependent pathway-including the PKA catalytic and regulatory subunits - have been characterized in P. falciparum, whether AKAPs are involved in this pathway remains unclear. Here, PfAKAL, an open reading frame of a potential AKAP-like protein in the P. falciparum genome was identified, and its protein partners and putative cellular functions characterized.

Methods: The expression of PfAKAL throughout the erythrocytic cycle of the 3D7 strain was assessed by RT-qPCR and the presence of the corresponding protein by immunofluorescence assays. In order to study physical interactions between PfAKAL and other proteins, pull down experiments were performed using a recombinant PfAKAL protein and parasite protein extracts, or with recombinant proteins. These interactions were also tested by combining biochemical and proteomic approaches. As phosphorylation could be involved in the regulation of protein complexes, both PfAKAL and Pf14-3-3l phosphorylation was studied using a radiolabel kinase activity assay. Finally, to identify a potential function of the protein, PfAKAL sequence was aligned and structurally modeled, revealing a conserved nucleotide-binding pocket; confirmed by qualitative nucleotide binding experiments.
\end{abstract}

Results: PfAKAL is the first AKAP-like protein in P. falciparum to be identified, and shares $23 \%$ sequence identity with the central domain of human AKAP18ס. PfAKAL is expressed in mature asexual stages, merozoites and gametocytes. In spite of homology to AKAP18, biochemical and immunochemical analyses demonstrated that PfAKAL does not interact directly with the P. falciparum PKA regulatory subunit (PFPKA-R), but instead binds and colocalizes with Pf143-31, which in turn interacts with PfPKA-R. In vivo, these different interactions could be regulated by phosphorylation, as PfPKA-R and Pf14-3-3l, but not PfAKAL, are phosphorylated in vitro by PKA. Interestingly, PfAKAL binds nucleotides such as AMP and CAMP, suggesting that this protein may be involved in the AMP-activated protein kinase (AMPK) pathway, or associated with phosphodiesterase activities.

Conclusion: PFAKAL is an atypical AKAP that shares common features with human AKAP18, such as nucleotides binding. The interaction of PfAKAL with PfPKA-R could be indirectly mediated through a join interaction with Pf143-31. Therefore, PfPKA localization could not depend on PfAKAL, but rather involves other partners.

Keywords: A-kinase anchoring protein like (AKAL), 14-3-3 protein, Plasmodium falciparum, AMP, Interactome, Nucleotide

\footnotetext{
*Correspondence: anais.merckx@parisdescartes.fr

${ }^{2}$ COMUE Sorbonne Paris Cité, Université Paris Descartes, Paris, France

Full list of author information is available at the end of the article
} 


\section{Background}

Malaria kills about 438,000 people per year, mostly in Africa [1]. Plasmodium falciparum, a protozoan parasite, is the deadliest of the five known species responsible for the disease in humans. Current malaria control strategies rely primarily on insecticide-treated bed nets and drugs. However, the extensive spread of drug resistance limits the armamentarium [2]. The identification and characterization of novel Plasmodium-specific proteins and their joint interaction may lead to the development of new therapeutic strategies.

Protein phosphorylation is one of the most common post-translational modifications involved in cellular communication. The phosphorylation status of a protein is determined by the balance between the activities of kinases and phosphatases. The cAMP-signalling pathway plays a central role in many developmental processes in eukaryotes, by activating molecules such as the cAMP-dependent protein kinase (PKA). A high concentration of cAMP interacting with regulatory subunits liberates catalytic subunits of PKA (PKA-C) to phosphorylate its target proteins. In eukaryotes, the subcellular specificity of PKA largely depends on its interaction with A-kinase anchoring proteins (AKAPs). This interaction ensures the spatio-temporal control of PKA activity, by tethering the enzyme close to its substrates, and by interacting with phosphatases and phosphodiesterases (PDE) [3-5].

AKAP proteins have a wide range of localizations, and can be associated with membranes, cellular organelles, and compartments. In the nucleus for instance, their localization is controlled through protein-lipid or protein-protein interactions [6]. The subcellular localization of AKAPs can be dynamic and regulated by phosphorylation [3]. Human AKAP18 is a low molecular weight AKAP with four different isoforms with distinct targeting domains. Because of their localizations, AKAP18 isoforms are involved in different cellular mechanisms. AKAP18 $\delta$, the larger isoform, has been reported to anchor PKA for both phosphorylation of aquaporin-2 in kidneys [4] and phosphorylation of phospholamban in the heart [5]. AKAP18 $\delta$ also binds PDE4A isoforms within its central region [6]. A common central domain of AKAP $18 \delta / \gamma$ has been described, whose structure resembles the $2 \mathrm{H}$ phosphoesterase family, harbouring a pair of conserved His-x-Thr motifs [7].

In $P$. falciparum, several effectors of the cAMP-pathways including cAMP-dependent protein kinase catalytic subunit (PKA-C) and regulatory subunit (PKA-R) homologues $[8,9]$, nucleotide cyclases $[10]$ and phosphodiesterase $[11,12]$ have been identified. Importantly, the PfPKA-C knock-out is lethal and the PfPKA-R overexpression inhibits the growth of the parasite suggesting a crucial role of these effectors in the development and survival of the parasite [9].

In this study, the expression and function of the $P$. falciparum protein encoded by PF3D7_0512900/PFE0640w [PlasmoDB.org], herein named PfAKAL ( $P$. falciparum A Kinase anchoring protein-like), based on its amino acid sequence homology with human AKAP18 [7], were investigated. However, unlike human AKAP18, PfPKA targeting and localization do not depend on direct binding to PfAKAL. This suggests that the subcellular localization of PfPKA may result from an alternative process and that the function of PfAKAL may differ from its human orthologs.

\section{Methods}

\section{RNA extraction and real-time quantitative PCR}

\section{experiments}

Plasmodium falciparum 3D7 strain cultures were synchronized twice with $5 \%$ D-sorbitol solution. The infected red blood cells were harvested every $8 \mathrm{~h}$ in order to have all the different intra-erythrocytic stages. Rings, early and late trophozoites, and schizont stages were harvested and conserved in Trizol. Stage-specific mRNA were extracted, and cDNA synthesized by RT-PCR. CGTAACGATGTTTTATAT (forward) and TAATATTTCTTTTGCGGG (reverse) primers specific to $140 \mathrm{pb}$ of the pfakal gene were designed. Realtime Quantitative PCR was performed on the cDNA to estimate pfakal expression levels throughout the intraerythrocytic development, using SensiFastSYBR NOROX Mix 2× (Bioline) and Rotor-gene ${ }^{\mathrm{TM}} 600$ (Corbett Research), and $0.208 \mu \mathrm{M}$ of each primer. The following PCR cycling conditions were used: initial heat activation step at $95^{\circ} \mathrm{C}$ for $1 \mathrm{~min}$, followed by 35 cycles of $95^{\circ} \mathrm{C}$ for $15 \mathrm{~s}, 60{ }^{\circ} \mathrm{C}$ for $1 \mathrm{~min}$ and $72{ }^{\circ} \mathrm{C}$ for $5 \mathrm{~min}$. All PCR reactions including negative controls (water) were carried out in duplicate, and a minimum of three experiments was carried out for each sample. The $p 90$ gene, encoding seryl-tRNA synthetase involved in DNA duplication was used as reference gene, and to characterize the RNA samples prior to the pfakal expression analysis. AAGTAGCAGGTCATCGTGGTT (forward) and TTCGGCACATTCTTCCATAA (reverse) primers specific to $158 \mathrm{pb}$ of the $p 90$ gene were designed. The amplification specificity for each primer pair was determined by melting-curve analysis of each PCR product. The pfakal transcript abundance was calculated using the $2^{-\Delta \Delta \mathrm{Ct}}$ method where $\mathrm{Ct}$ is the threshold cycle, and $\Delta \Delta \mathrm{Ct}=$ $(\mathrm{Ct} p f a k a l-\mathrm{Ct} p 90)_{\text {stage }}-(\mathrm{Ct} p f a k a l-\mathrm{Ct} p 90)_{\text {reference stage }}$. The result for each sample was expressed by calculating the mean and standard deviations between the three experiments. A statistical analysis was performed with a Kruskal-Wallis test. 


\section{Molecular cloning and bacterial expression of recombinant proteins}

Oligonucleotides were designed for amplification of a 798 bp region on chromosome 5, carrying the pfakal coding sequence and cloning into pGEX-6P1 (forward primer/BamH1, cgGGATCCATGAATATTAAAAGAAG CATATACCA; reverse primer/Xho1, cgCTCGAGTC AAAGATTAAACTCAGACACG) and pRSF Duet (forward primer/BamH1, GGATCCAATGAATATTAAAAG AAGCATATACCATTATTTAAG; reverse primer/Not 1 : GCGGCCGCTCAAAGATTAAACTCAGACACGATT T). Pf14-3-3I [PlasmoDB: MAL8P1.69/PF3D7_0818200] and PfPKA-R [PlasmoDB: PFL1110c/PF3D7_1223100] genes were also amplified by PCR prior cloning into pRSFDuet vector (forward pf14-3-3I primer/EcoRI: GAATCC GATGGCAACATCTGAAGAATT; reverse pf14-3-3I primer/Not1 GCGGCCGCTCATTCTAATCCTTCGTC; forward pfpka-r primer/EcoRI GAATTCGATGGGCA ATGTGTGCAC; reverse $p f p k a-r$ primer/Not 1 GCGGCC GCTTAAT T T TCATCAATACAAGTTGTATCCA). After amplification from a $P$. falciparum cDNA library, the PCR products were digested prior insertion in the plasmids. For the different cloning steps, the inserts were verified by sequencing, and the plasmids were separately transformed into Escherichia coli BL21 Codon Plus (Stratagene) cells.

GST-PfAKAL, (His)6-PfPKA-R and (His) 6 -Pf14-3-3I protein expressions were induced for 4 and $6 \mathrm{~h}$ with 0.3 and $0.5 \mathrm{mM}$ of Isopropyl $\beta$-D-1-thiogalactopyranoside (IPTG) at $37{ }^{\circ} \mathrm{C}$, respectively. (His) $6_{6}$ PfAKAL expression was induced overnight with $0.3 \mathrm{mM}$ of IPTG at $20{ }^{\circ} \mathrm{C}$. All purification steps were performed at $4{ }^{\circ} \mathrm{C}$. The bacterial pellets were lysed with lysosyme and by sonication in lysis buffer with ComplexTM mixture protease inhibitor tablet from Roche Applied Science. (GST-PfAKAL lysis buffer: $1 \times$ PBS, pH 7.5, $0.1 \%$ Triton, 1 mM EDTA; (His) ${ }_{6}$-PfAKAL, (His) ${ }_{6}$-PF14-3-3I and (His) ${ }_{6}$-PfPKA-R lysis buffer: $20 \mathrm{mM}$ Tris- $\mathrm{HCl} \mathrm{pH} 8,300 \mathrm{mM} \mathrm{NaCl}, 1 \%$ Triton $\times 100$ ). The lysates were cleared by centrifugation (9000 rpm at $4{ }^{\circ} \mathrm{C}$ for $1 \mathrm{~h}$ ), and the soluble fraction was incubated for $1.5 \mathrm{~h}$ using mild agitation with glutathioneagarose (Sigma-Aldrich) or Ni-NTA-agarose (Invitrogen) resin.

GST-PfAKAL slurry was washed in lysis buffer, and the fusion protein eluted with elution buffer (30 mM Tris, $\mathrm{pH}$ 8.0, $15 \mathrm{mM} \mathrm{NaCl}, 20 \mathrm{mM}$ glutathione). (His) 6 tagged protein slurry was washed three times in lysis buffer, twice in lysis buffer with $20 \mathrm{mM}$ imidazole, and eluted with elution buffer (lysis buffer with $250 \mathrm{mM}$ Imidazole). All recombinant protein preparations were confirmed by anti-GST or anti-(His) ${ }_{6}$ western blot analysis.

\section{Gel filtration analysis}

To determine if proteins are forming dimer, gel filtration analysis were performed on the eluted fractions obtained after affinity purification of (His) ${ }_{6}$-Pf14-3-3I and (His) ${ }_{6}$ PfPKA-R. Samples were loaded onto a Superdex S200 10/300 Increase column (GE Healthcare) and gel filtration were carried out using GF buffer $(20 \mathrm{mM}$ Tris $-\mathrm{HCl}$ at $\mathrm{pH} 8,300 \mathrm{mM} \mathrm{NaCl}$ ). The fractions corresponding to the pic detected by UV were analysed by a $12.5 \%$ SDSPAGE to confirm the size of the eluted proteins.

\section{Immunofluorescence assay (IFA)}

In vivo immunizations were carried out on 6-week old Swiss female mice (Janvier, Le Genest De L'Isle, France). Four mice were first immunized by an intraperitoneal injection of $10 \mu \mathrm{g}$ of recombinant PfAKAL fused to a GST tag (produced as described above) homogenized in $1 \mathrm{mg}$ aluminium hydroxide (Alugel, Serva) in a $100 \mu \mathrm{l}$ saline volume. Two boosts of $10 \mu \mathrm{g}$ of recombinant PfAKAL fused to a (His) ${ }_{6-}$ tag homogenized in $1 \mathrm{mg}$ aluminium hydroxide in a $100 \mu \mathrm{l}$ saline volume were administered on days 21 and 42. Blood samples were collected by retro-orbital bleeding at day 56. Rat Anti Pf14-3-3I antibodies were obtained according to [13].

Plasmodium falciparum 3D7 strain infected red blood cells with $7 \%$ parasitaemia (mostly trophozoites and schizonts) were washed in PBS. The cells were fixed with methanol. After overnight incubation in PBS, $2 \%$ BSA the blood smears were incubated with one of the following antisera: anti-PfAKAL mouse antiserum diluted 1:500 and anti-Pf14-3-3I rat antibodies diluted 1:100, or an anti-PfAMA1/28G2d described in [14] and diluted 1/1.000 (kindly provided by J.C. Barale, Institut Pasteur, Paris). After washes, the slides were incubated with AlexaFluor conjugated secondary antibodies against mouse or rat IgG containing 1/20,000 Hoechst (Hoechst 33,342, trihydrochloride, trihydrate $10 \mathrm{mg} / \mathrm{mL}$ ) and mounted in vectashield (Vectorslab). Samples were observed at $100 \times$ magnification using an Olympus fluorescent microscope. Thereafter, the PfAKAL and Pf14-3-3I cellular localizations were analysed using the Imaris deconvolution software from Bitplane.

\section{Peptide array experiment}

128 overlapping 15 mer-peptides with a two amino acids frameshift, derived from the full-length PfAKAL amino acid sequence were synthesized on nitrocellulose membrane. The membrane was incubated in a $1 \mu \mathrm{M}$ (His) ${ }_{6}$-PfPKA-R protein recombinant solution. An antipolyhistidine western blot was performed to identify the peptides having interacted with (His) ${ }_{6}$-PfPKA-R. 
Pull-down experiments with parasite protein extracts Plasmodium falciparum (3D7) pellets were sonicated in RIPA buffer (30 mM Tris, pH 8.0, $150 \mathrm{mM} \mathrm{NaCl}, 20 \mathrm{mM}$ $\mathrm{MgCl}_{2}, 1 \mathrm{mM}$ EDTA, $1 \mathrm{mM}$ dithiothreitol, $10 \mu \mathrm{M}$ ATP, 0.5 \% Triton X-100, 1 \% Nonidet P-40, Complete EDTA free protease inhibitors Coktail (Roche), and PhosSTOP Phosphatase inhibitors Coktail (Roche). The lysates were cleared by centrifugation $\left(15,000 \mathrm{rpm}\right.$ for $30 \mathrm{~min}$ at $\left.4{ }^{\circ} \mathrm{C}\right)$, and the total amount of protein in the supernatant was measured using a Coomassie Plus Protein Assay Reagent (Pierce). The beads coated with GST, GST-PfAKAL or AMP were incubated with the parasite extracts or in RIPA buffer alone at $4{ }^{\circ} \mathrm{C}$, under mild agitation for $90 \mathrm{~min}$ $(100 \mu \mathrm{g}$ of total parasite proteins for $10 \mu \mathrm{g}$ of recombinant protein on beads). The beads were then washed three times in RIPA buffer, and once in RIPA buffer with $0.1 \%$ SDS. The precipitated complexes were analysed by mass spectrometry (Proteomic Facility, 3P5 Université Paris Descartes).

\section{Protein/protein and protein/nucleotide interaction experiments}

GST, GST-PfAKAL glutathione beads were incubated with (His) ${ }_{6}$-Pf14-3-3I or (His) ${ }_{6}$-PfPKA-R recombinant proteins. Also, GST and GST-PfPKA-R glutathione beads were incubated in (His) ${ }_{6}$-Pf14-3-3I recombinant protein. Adenosine monophosphate (AMP) or cyclic AMP crosslinked agarose beads were incubated in recombinant (His) 6 -PfAKAL protein solution for $2 \mathrm{~h}$ at $4{ }^{\circ} \mathrm{C}$.

For all pull down experiments, beads were washed in RIPA buffer, and the protein complexes were analysed by SDS-PAGE and an anti-histidine western blot (Sigma Aldrich Monoclonal Anti-polyHistidine Peroxidase Conjugate) was performed to identify interactions.

\section{Kinase assay}

Assays were performed in a standard reaction $(30 \mu \mathrm{l})$ containing $50 \mathrm{mM}$ MOPS pH7, $0.5 \mathrm{mM} \mathrm{MgCl}_{2}, 15 \mu \mathrm{M}$ ATP, $5 \mu \mathrm{Ci}$ of $[\gamma-32 \mathrm{P}]$ ATP $(3000 \mathrm{Ci} / \mathrm{mmol}$; Perkin Elmer $)$ and $1 \mu \mathrm{M}$ of recombinant (His) ${ }_{6}$-PfPKA-R, $1 \mu \mathrm{M}$ recombinant (His) ${ }_{6}$-Pf14-3-3I or $1 \mu \mathrm{M}$ recombinant (His) ${ }_{6}$ PfAKAL. The reactions were initiated by addition of $1 \mu \mathrm{M}$ commercial bovine PKAc (Sigma Aldrich P2645), with or without $1 \mu \mathrm{M} \mathrm{H} 89$, a potent PKA inhibitor. The kinase reactions, proceeded for $30 \mathrm{~min}$ at $30{ }^{\circ} \mathrm{C}$, were stopped by the addition of Laemmli buffer, boiled for 3 min and analysed by electrophoresis on $8 \%$ SDS-polyacrylamide gel. The gels were dried and submitted to autoradiography.

PfAKAL structural model and binding site predictions Sequence alignments of PfAKAL to Plasmodium yoelii AKAP18 [PlasmoDB: PY04627], a Plasmodium vivax ortholog [PlasmoDB: PVX080595] and human AKAP18 $\gamma$ isoform were performed using ClustalO, and visualized using BOXSHADE. Amino acid sequences of PfAKAL was submitted to the I-TASSER server for structural prediction $[15,16]$. Protein structures were visualized using PyMol version 0.99rc6.

\section{Results and discussion}

In silico identification of a $P$. falciparum ORF related to an AKAP

BLASTP searches of the PlasmoDB database were performed, using as queries a variety of AKAP sequences from different organisms, identifying a single $P$. falciparum polypeptide displaying significant amino acid homology. The predicted 265-residue protein, PfAKAL [PlasmoDB PFE0640w/PF3D7_0512900] displays $23 \%$ identity, and $33 \%$ similarity to human AKAP18y. The parasite protein also shows homology to predicted proteins from other Plasmodium species, such as 50 and $44 \%$ identities with P. yoelii [PlasmoDB: PY04627], and P. vivax [PlasmoDB: PVX080595], respectively (Fig. 1). However, PfAKAL does not share all of the characteristics typical of mammalian AKAPs, such as, for example, a transmembrane domain, N-myristoylation, or palmytoylation sites suggesting that PfAKAL is cytosolic as are human AKAP18 $\delta$ and AKAP18y [17]. In addition to its homology with the core domain of human AKAP18 $\gamma$, PfAKAL contains a Pfam AKAP7 $2^{\prime} 5^{\prime}$ RNA ligase-like domain (pfam10469). This domain corresponds to the N-terminal part of AKAP7 which is known to play a role in regulating PKA-mediated gene transcription in somatic cells and oocytes [17]. PfAKAL also harbours two His-x-Thr/Ser motifs (Fig. 1) that define the $2 \mathrm{H}$ phosphoesterase family, including RNA ligases and cyclic nucleotide phosphoesterases [18]. Such residues are conserved in the AKAP18 $\delta / \gamma$ from different organisms [7]. Despite the weak amino acid homology, PfAKAL is the only AKAP-like protein to be identified in the predicted proteome of $P$. falciparum and its role remains unknown.

\section{PfAKAL expression and localization throughout the erythrocytic stage}

To determine whether pfakal is expressed during red blood cell infection, real-time quantitative PCR (RTqPCR) experiments were performed on total cDNA from synchronized parasite erythrocytic stages from in vitro cultures of the 3D7 strain [19]. Primers efficiency were validated at 1.98 and 1.93 for $p 90$ and pfakal gene respectively, and a Kruskal-Wallis test was performed on the results, with a $p$ value $=0.0077$. The pfakal gene is expressed throughout the erythrocytic cycle, with maximal expression in mature trophozoite stages (Fig. 2), thus the expression levels were normalized to pfakal gene 


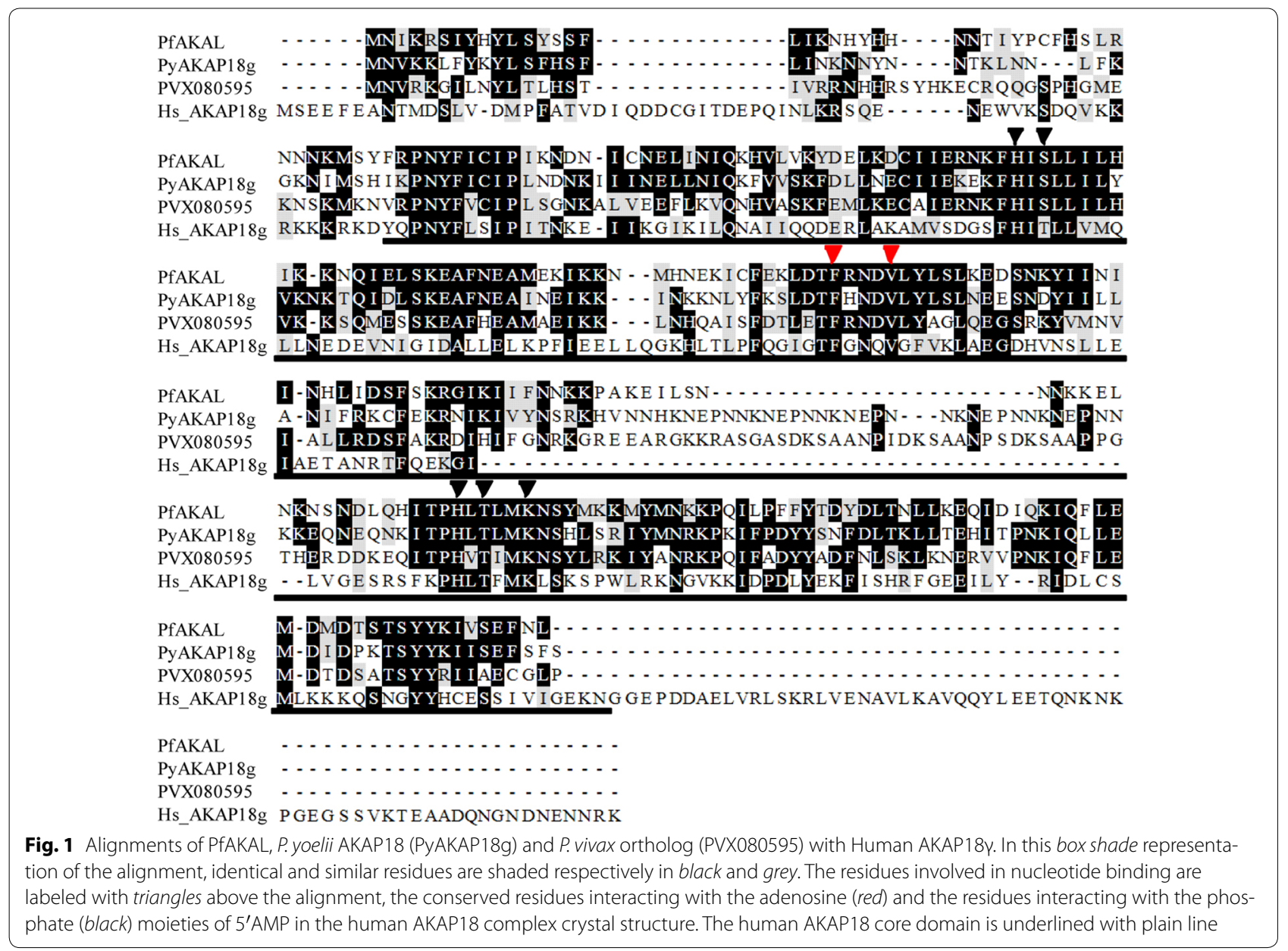

transcription level in late trophozoites (reference stage in $\Delta \Delta \mathrm{Ct}$ equation). The results correspond with those previously described in a large scale study [20].

To analyse the subcellular distribution and stage specificity of PfAKAL expression, immunofluorescence assays were carried out on $P$. falciparum-infected erythrocytes harbouring parasites at different development stages (Fig. 3). In asexual parasites PfAKAL was undetectable in ring stages (Fig. 3a), with expression predominantly in late trophozoites and schizonts. Interestingly, a diffuse subcellular localization of PfAKAL was observed in the parasite cytoplasm and in the nucleus in trophozoite stages, whereas it was mostly nuclear in schizonts (Fig. 3b, c). In sexual stages, PfAKAL was also detected in the cytoplasm of the parasite, although it mainly accumulates around the nucleus (Fig. $3 \mathrm{~d}-\mathrm{g}$ ). To analyse the PfAKAL localization on merozoïtes immunofluorescence assays were also performed with a dual labelling using mouse anti-PfAKAL antibody with a rat anti-PfAMA1, a control labelling parasite plasma membrane. PfAKAL shares partial location with PfAMA1, and the IFA results showed that PfAKAL is expressed in egressing (Fig. 4a) and invading (Fig. 4b) merozoites, in agreement with a wide proteomic analysis of P. falciparum cycle [21].

These results strongly suggest that PfAKAL is expressed at the transcript level as well at the protein level during the intra-erythrocytic development of P. falciparum parasites, but PfAKAL remains within the parasite and is not exported to the infected red blood cell cytosol. The data is consistent with the in silico analysis suggesting that PfAKAL is not a membrane associated protein.

\section{PfPKA anchoring does not depend on direct interaction with PfAKAL}

In human cells, AKAPs bind PKA through an amphipathic $\alpha$-helical structure consisting of 14-18 amino acids (RII-binding domain), which interacts with the hydrophobic groove formed by the N-terminal dimerization and docking (D/D) domain of PKA-R subunits [22]. To investigate the potential interaction between PfAKAL and PfPKA regulatory subunit, recombinant proteins were produced and used for GST pull-down assays. 


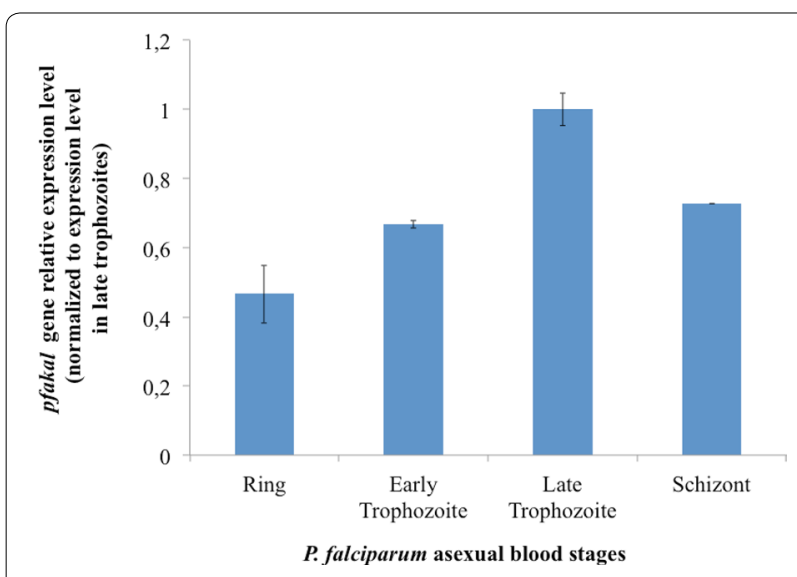

Fig. 2 Pfakal expression levels in infected red blood cells. RT-qPCR analyses were performed on synchronized $P$. falciparum asexual blood stages. The pfakal transcript abundance was calculated using the $2^{-\Delta \Delta \mathrm{Ct}}$ method where $\mathrm{Ct}$ is the threshold cycle, and $\Delta \Delta \mathrm{Ct}=(\mathrm{Ct} p f a k a l-\mathrm{Ct} p 90)_{\text {stagex }}-(\mathrm{Ct} \text { pfakal }-\mathrm{Ct} p 90)_{\text {late trophozoite. }}$ Expression levels were normalized to pfakal gene transcription level in late trophozoites, the maximal expression observed throughout the erythrocyte cycle. The result for each sample was expressed by calculating the mean and standard deviations (bars on histograms) between the three experiments. A statistical analysis was performed with a Kruskal-Wallis test $(p=0.0077)$

Purified GST-PfPKA-R beads were incubated in purified (His) ${ }_{6}$-PfAKAL solution. Anti-polyhistidine western blot analysis failed to reveal any interaction between the two proteins, suggesting that there is no direct interaction between the two proteins (Fig. 5a, b, lane 8). The ability of PfAKAL to bind PfPKA-R was also investigated using a peptide array analysis. To this end, recombinant (His) ${ }_{6}$-PfPKA-R was incubated with a membrane containing PfAKAL derived peptides and again, anti-polyhistidine immunoblot analysis did not reveal the presence of (His) ${ }_{6}$-PfPKA-R on the membrane. Thus, this particular array did not reveal any interaction between the two proteins. The two positive dots correspond to a positive control peptide (containing six histidines, bottom line) and a derived peptide from PfAKAL containing three histidines (top line) (Fig. 5c).

Even though peptide array technology has been used successfully to study PKA-R/AKAP interaction in other organisms [23, 24], the fact that a 15-mer peptide doesn't mimic the complete docking site cannot be excluded, nor the possibility of peptide and PfAKAL or PfPKA-R recombinant protein mis-folding.

Therefore, to determine if PfPKA might be an interacting partner of PfAKAL in the parasite, pull-down experiments using recombinant GST-PfAKAL and native parasite protein extracts were performed and followed by mass spectrometry nLC-MSMS analysis. This approach confirmed the absence of any direct interaction between PfAKAL and PfPKA-R.

Furthermore, an In silico analysis of PfPKA-R highlighted a difference of the $\mathrm{N}$-terminal portion compared to the equivalent $\mathrm{N}$-terminal part of human PKA-R, as it does not share the prototypical helical bundle that allows dimerization of PKA-R subunits, and the docking of AKAP proteins [25]. An analytical gel filtration analysis on (His) ${ }_{6}$-PfPKA-R was also performed, and showed only one pic (Fig. 5d). This pic corresponds to a monomer of (His) ${ }_{6}$-PfPKA-R, according to the calibration of the Superdex S200 10/300 Increase column (GE Healthcare) around a molecular weight of $50 \mathrm{kDa}$. The eluted fractions from the pic were collected and analysed by $12.5 \%$ SDS-PAGE to confirm that the pic corresponds to (His) ${ }_{6}$-PfPKA-R. These observations are in agreement with the lack of direct interaction between PfAKAL and PfPKA-R, and raise the hypothesis of an alternative way of PKA anchoring in the malaria parasite. This hypothesis was recently reinforced by the antimalarial activity of a human AKAP disruptor peptide STAD-2, through a PKA-independent mechanism [26], and by a recent analysis highlighting the fact that AKAP can only be found in animal genomes, and likely evolved in conjunction with multicellularity [27]. Several PKA phosphorylation sites have however been identified in the N-terminal part of PfPKA-R [28-30], and Haste and colleagues suggested that these sites might be involved in the docking of other proteins such as Pf14-3-3 [25].

\section{PfAKAL/PfPKA complex might depend on Pf14-3-3I}

The combination of pull-down experiments with GSTPfAKAL beads incubated with parasite protein extracts and nLC-MSMS analysis identified Pf14-3-3I [PlasmoDB: MAL8P1.69/PF3D7_0818200] [13], as a putative PfAKAL partner. We further investigated this interaction with GST-PfAKAL and (His) ${ }_{6}$-Pf14-3-3I recombinant proteins. Purified GST-PfAKAL beads were incubated in purified (His) 6 $_{6}$ Pf14-3-3I solution, and binding analysed by anti-polyhistidine western blot. As expected, GSTPfAKAL bound (His) ${ }_{6}$-Pf14-3-3I, while GST alone did not (Fig. 6a). To confirm this interaction, co-immunofluorescence assays were performed on infected red blood cells with anti-PfAKAL and anti-Pf14-3-3I antibodies. Pf14-3-3I is known to be present in both cytoplasmic and nuclear compartments of the parasite [13], but these locations seem more diffuse than that of PfAKAL throughout the parasite development. The distribution of the two proteins partially overlapped in the parasite cytoplasm and nucleus (Fig. 6b). Partial co-localization was confirmed following deconvolution of the IFA 


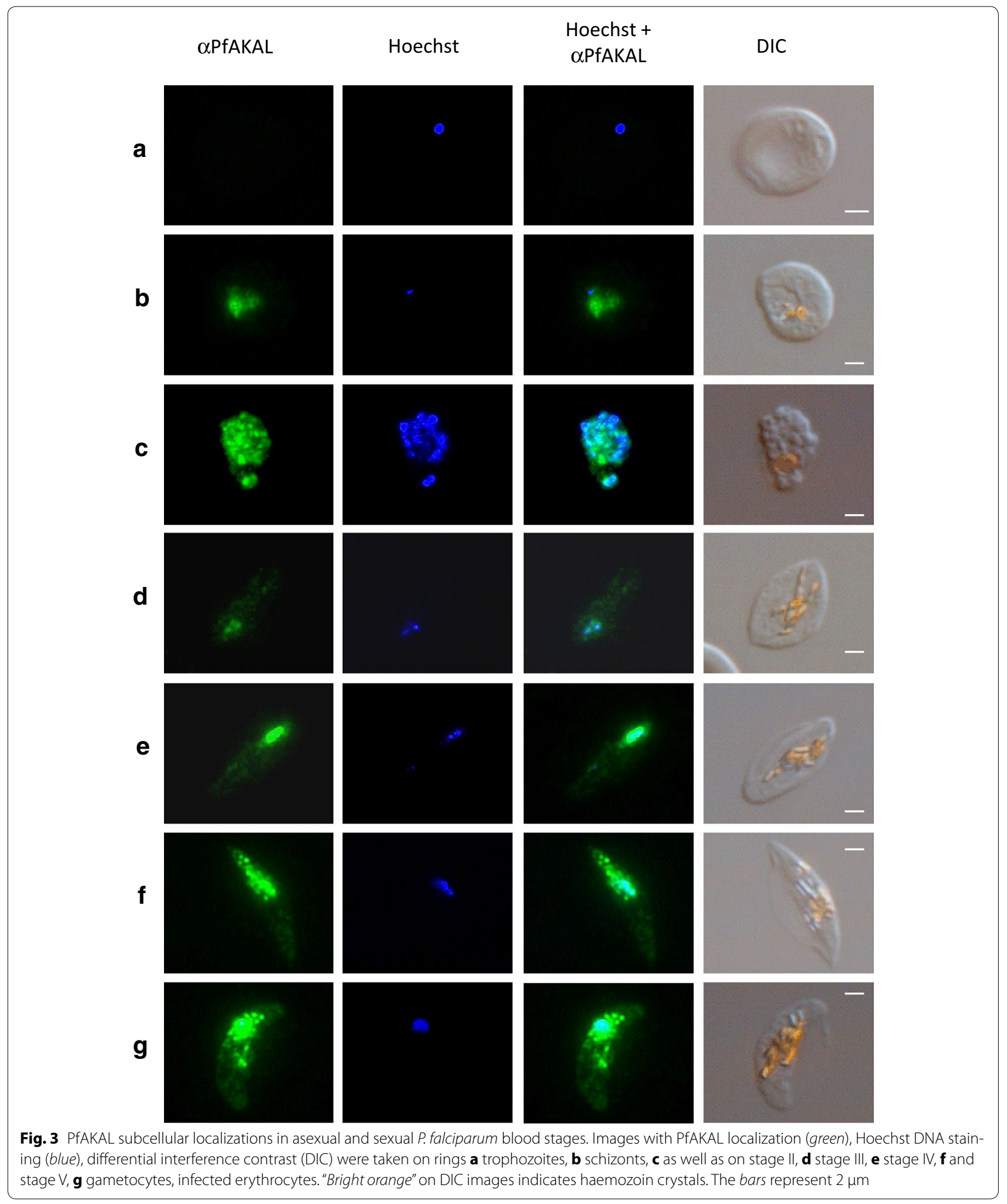

images (Fig. 6c). When combined with pull-downs, the IFA images confirm the interaction between PfAKAL and Pf14-3-3I.
14-3-3 proteins are known to play scaffold functions in cells to stabilize multiple enzyme complexes including PKA subunits [31], and to be phosphorylated by 
QPfAKAL $\quad$ PPfAMA1 Hoechst $\alpha$ PfAMA1 $+\alpha \operatorname{PfAKAL}$

\section{DIC}
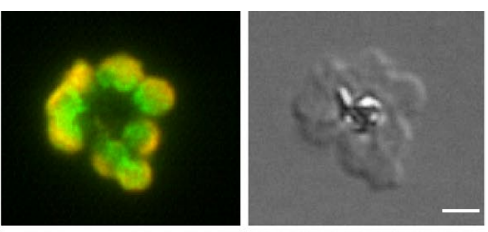

a
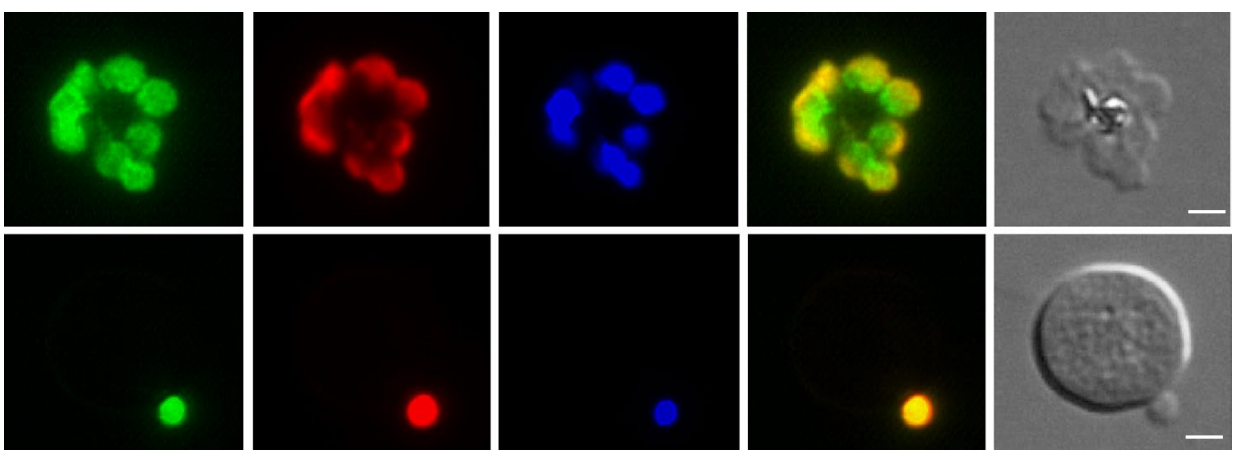

Fig. 4 PfAKAL localization in P. falciparum merozoites by indirect immunofluorescence assays. Images with PfAKAL localization (green), PfAMA1 localization (red), Hoechst DNA staining (blue) and differential interference contrast (DIC) and were taken. a P. falciparum 3D7 egressing merozoites, b P. falciparum 3D7 invading merozoite. The bars represent $2 \mu \mathrm{m}$
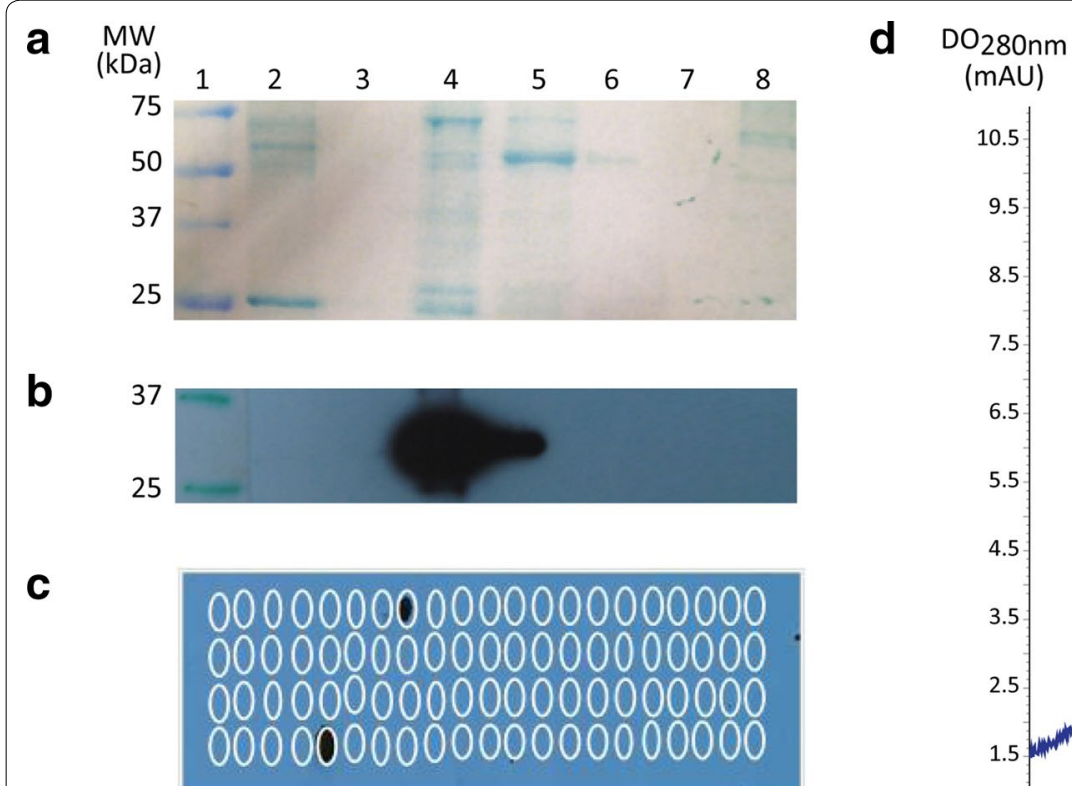

(mAU)

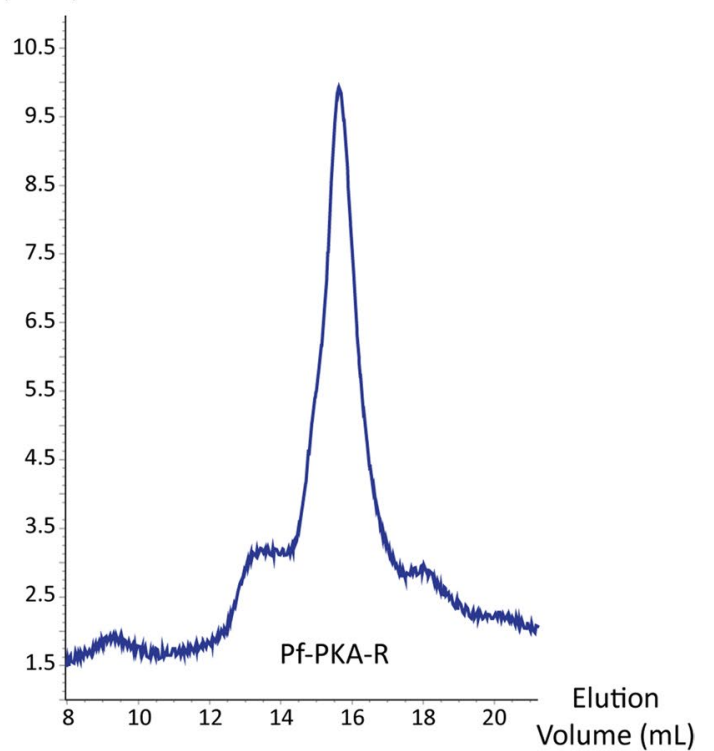

Fig. 5 Interaction between GST-PfPKA-R and (His) $)_{6}$ PfAKAL protein. a Nitrocellulose membrane coloration and anti-polyhistidine Western blot (b) after GST beads (2) and GST-PfPKA-R beads (8) were incubated in (His) $6_{6}$ PfAKAL solution. Molecular marker (1), empty lane (3), (His) 6 -PfAKAL (4), flowthrough after incubation of GST-PfPKA-R beads in (His) ${ }_{6}$-PfAKAL solution (5), beads washes (6-7). The recombinant GST-PfPKA-R and (His) ${ }_{6}-$ PfAKAL proteins molecular weight are 75 and $32 \mathrm{kDa}$ respectively. c A peptide array was performed using peptides derived from the PfAKAL whole protein sequence. An anti-polyhistidine western blot was realized to identify the peptides interacting with (His) ${ }_{6}$-PfPKA-R. d Gel filtration analysis of (His) 6 -PfPKA-R

cAMP-dependent kinases [32]. Therefore the interaction between Pf14-3-3I and PfPKA-R was investigated by pulldown experiments involving recombinant proteins. Purified GST-PfAKAL, GST-PfPKA-R and GST (as control) beads were incubated in purified (His) ${ }_{6}$-Pf14-3-3I solution, and the complexes analysed by anti-polyhistidine western blot (Fig. 6d). Both PfAKAL (Fig. 6d, lane 4) and PfPKA-R (Fig. 6d, lane 5) interact with Pf14-3-3I suggesting that Pf14-3-3I could mediate binding between the two proteins acting as a scaffold in the assembly of a PfPKA/PfAKAL complex. As PfPKA-R binds Pf14-3-3I it also suggests that Pf14-3-3I might be phosphorylated by 


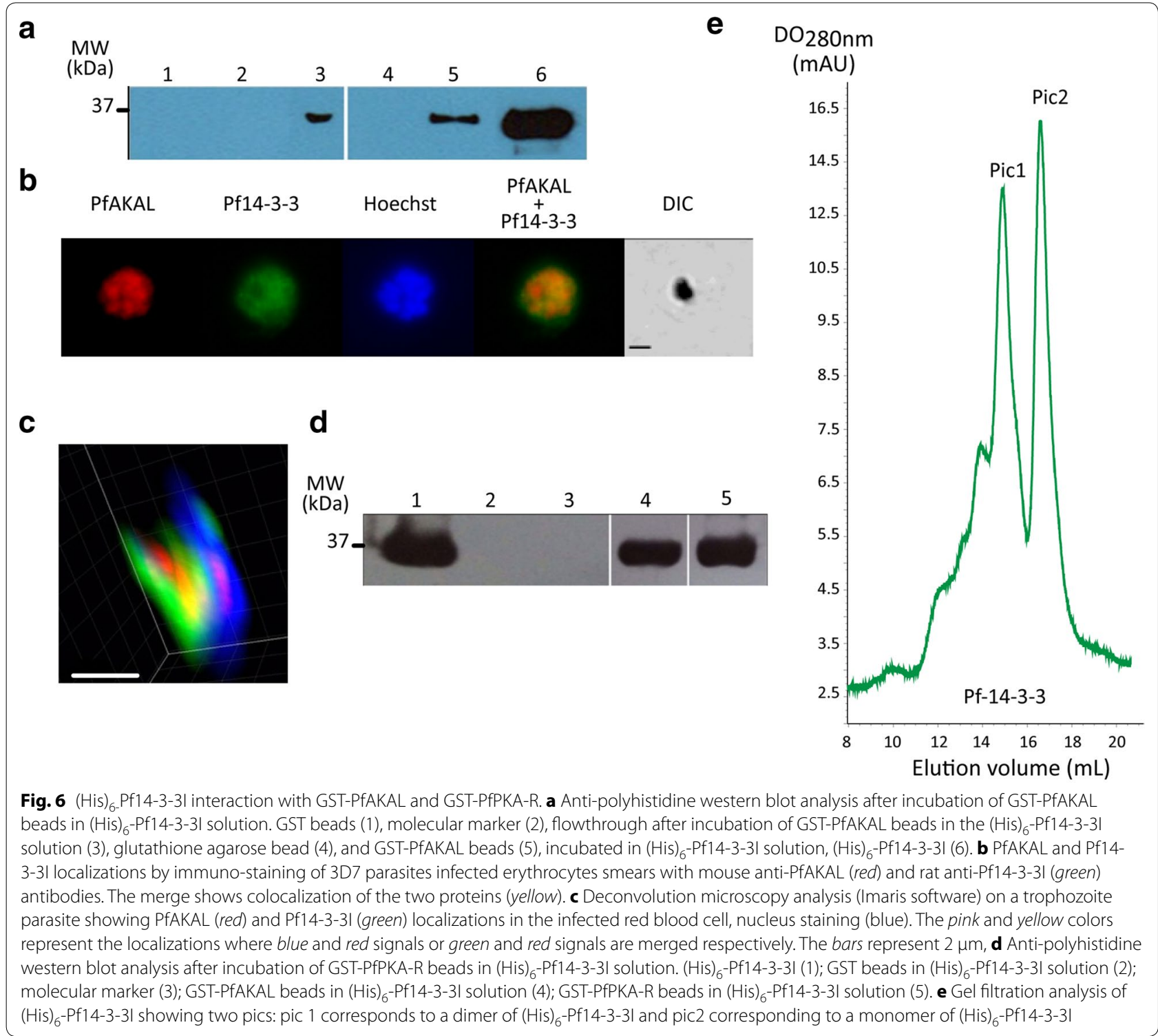

PfPKA-C, as phosphorylation of 14-3-3 by PKA has been reported to play a role in the regulation of the dimerization of 14-3-3 [32]. An analytical gel filtration performed on (His) 6 -Pf14-3-3I samples demonstrated the dimerization of Pf14-3-3I, as two pics were obtained on Superdex S200 10/300 Increase column (GE Healtcare), corresponding to the size of a dimer (around $62 \mathrm{kDa}$ ) and a monomer of (His) 6 -Pf14-3-3I (around $31 \mathrm{kDa}$ ) (Fig. 6d). The nature of the proteins in the two eluted fractions was confirmed by $12.5 \%$ SDS-PAGE analysis.

\section{PKA phosphorylates PfPKA-R and Pf14-3-3I}

First, the PfAKAL protein sequence was analysed with an algorithm predicting PKA phosphorylation potential sites [33] and identified a single site with a very weak score. To investigate the potential phosphorylation of PfPKA-R and Pf14-3-3I, an in vitro phosphorylation assay was performed with recombinant proteins (His) ${ }_{6}$ Pf14-3-3I, (His) ${ }_{6}$-PfPKA-R and (His) ${ }_{6}$-PfAKAL. Plasmodium falciparum (PfPKA-C) and bovine PKA catalytic subunits (PKA-C) share $48 \%$ similarity in the overall primary sequence, but $80 \%$ similarity in the activation segment that interacts with the substrate [34]. PKA phosphorylation sites also share a consensus sequence, R-R-X-S/T- $\Phi$, where $\Phi$ represents a hydrophobic residue. Therefore bovine PKA catalytic subunit could be used in our kinase assays, to assess the phosphorylation of the different proteins. The results showed the 
phosphorylation of PfPKA-R and Pf14-3-3I by bovine PKA, and that these phosphorylations are inhibited by the potent PKA inhibitor H89 (Fig. 7). On the contrary, PfAKAL was not phosphorylated in vitro by bovine PKA (Fig. 7). The results confirmed the prediction of a weak probability for PfAKAL to be phosphorylated by PKA. Also, the PKA phosphorylation of PfPKA-R and Pf143-3I were reinforced by $P$. falciparum schizont phosphoproteome studies $[29,30]$.

As 14-3-3 proteins act as chaperones and bind actin to promote nuclear import or export [35, 36], Pf14-3-3I could play a role in the localization of PfAKAL, depending on the PKA-dependent phosphorylation state of Pf143-3I. This hypothesis needs to be further investigated.

\section{In silico structural analysis of PfAKAL protein reveals conservation of residues responsible for nucleotide binding}

To determine the potential function of PfAKAL, the amino acid sequence was submitted to the I-TASSER server for protein structure prediction $[15,16]$. The returned sequence alignments and structural analogues were human AKAP18, bacterial 2'-5' RNA-ligase, cyclic nucleotide phosphodiesterase from Arabidopsis thaliana. The I-TASSER server also predicted five structural models for PfAKAL. The backbone chains of all five structural models share the same general shape, but differ in some secondary structures. In the highest scoring model, the $\mathrm{N}$-terminus is folded in an alpha-helix, but its conformation and location are different from that observed in the other models, and a binding pocket is present. The back of this pocket is lined by two $\beta$-sheets, whereas there is only one $\beta$-sheet in the other models (Fig. 8a). This highest scoring model is displayed alongside the structure of human AKAP18 co-crystallized with cytidine$5^{\prime}$-monophosphate (5'CMP) [7]. A superposition of PfAKAL structural model and human AKAP18 core domain structure confirmed that the two proteins share a similar shape (Fig. 8a). I-TASSER also predicted some binding sites [37] using human AKAP18 core domain and two phosphodiesterases from Arabidopsis thaliana and mouse. The highest scoring model, containing the predicted binding site from human AKAP18, highlights a possible interaction between PfAKAL and nucleotides such as AMP. The residues involved in AMP binding are conserved, but with some modifications in the orientation of the side chains (Fig. 8b).

A previous study also suggested that human AKAP18 acts as an adenosine monophosphate (AMP) sensor in the cell, binding AMP within a groove, located between the two lobes of the protein, and specifically binding $5^{\prime} \mathrm{AMP}$ and $5^{\prime} \mathrm{CMP}$ [7]. To investigate the nucleotidebinding capability of PfAKAL with $5^{\prime} \mathrm{AMP}$ and $3^{\prime}-5^{\prime}$ cAMP, pull-down experiments were performed with 5'AMP immobilized on agarose beads in a solution of recombinant PfAKAL or in parasite protein extracts. Western blot analyses showed that recombinant (His) ${ }_{6}-$ PfAKAL interacts with 5'-AMP (Fig. 8c).

A mass spectrometry analysis of the native complexes from parasite protein extracts interacting with AMPbeads also confirmed that native PfAKAL is a $5^{\prime}$-AMP binder. The binding of $5^{\prime}$-AMP did not interfere with the interaction between PfAKAL and Pf14-3-3I (Fig. 8d). AMP binding by PfAKAL suggests different functions

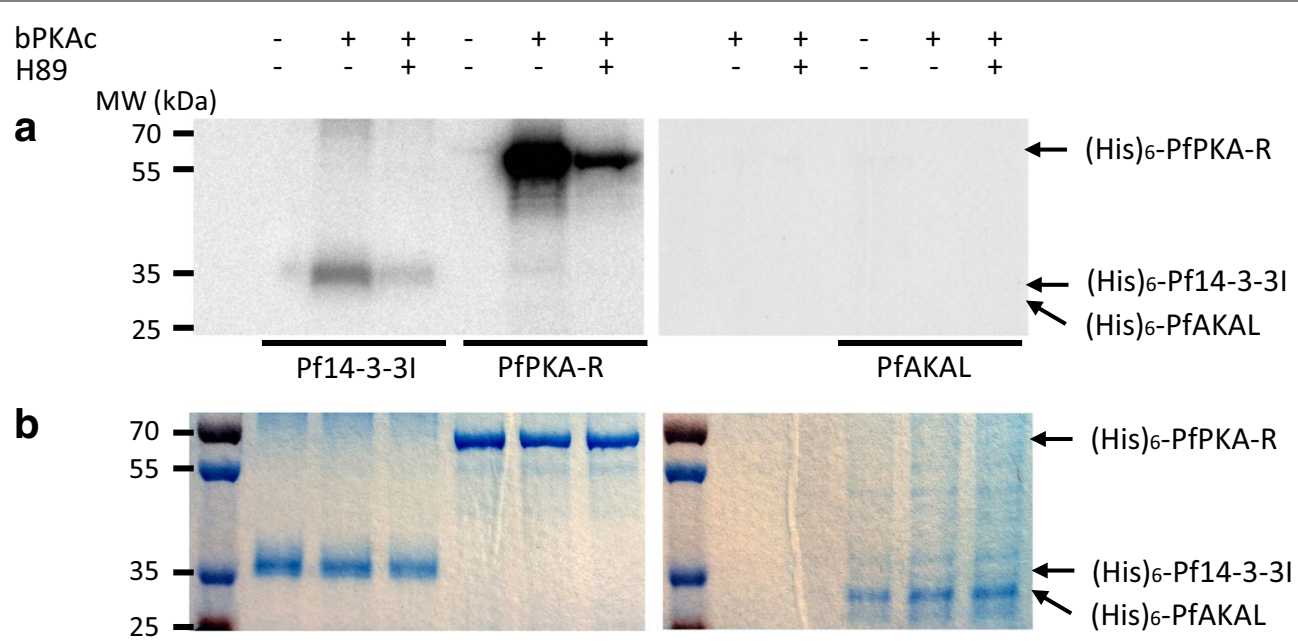

Fig. 7 Phosphorylation of (His) $)_{6}$ PfPKA-R, (His) $)_{6}$ Pf14-3-3l or (His) 6 -PfAKAL by bovine PKA catalytic subunit (bPKA). Radiolabel kinase activity assays were deployed to detect phosphorylation of the proteins. Assays without PKA or with H89, a potent PKA inhibitor were used as negative controls. Coomassie blue staining $(\mathbf{b})$ of radiolabelled SDS-gels (a) was used as a loading control 


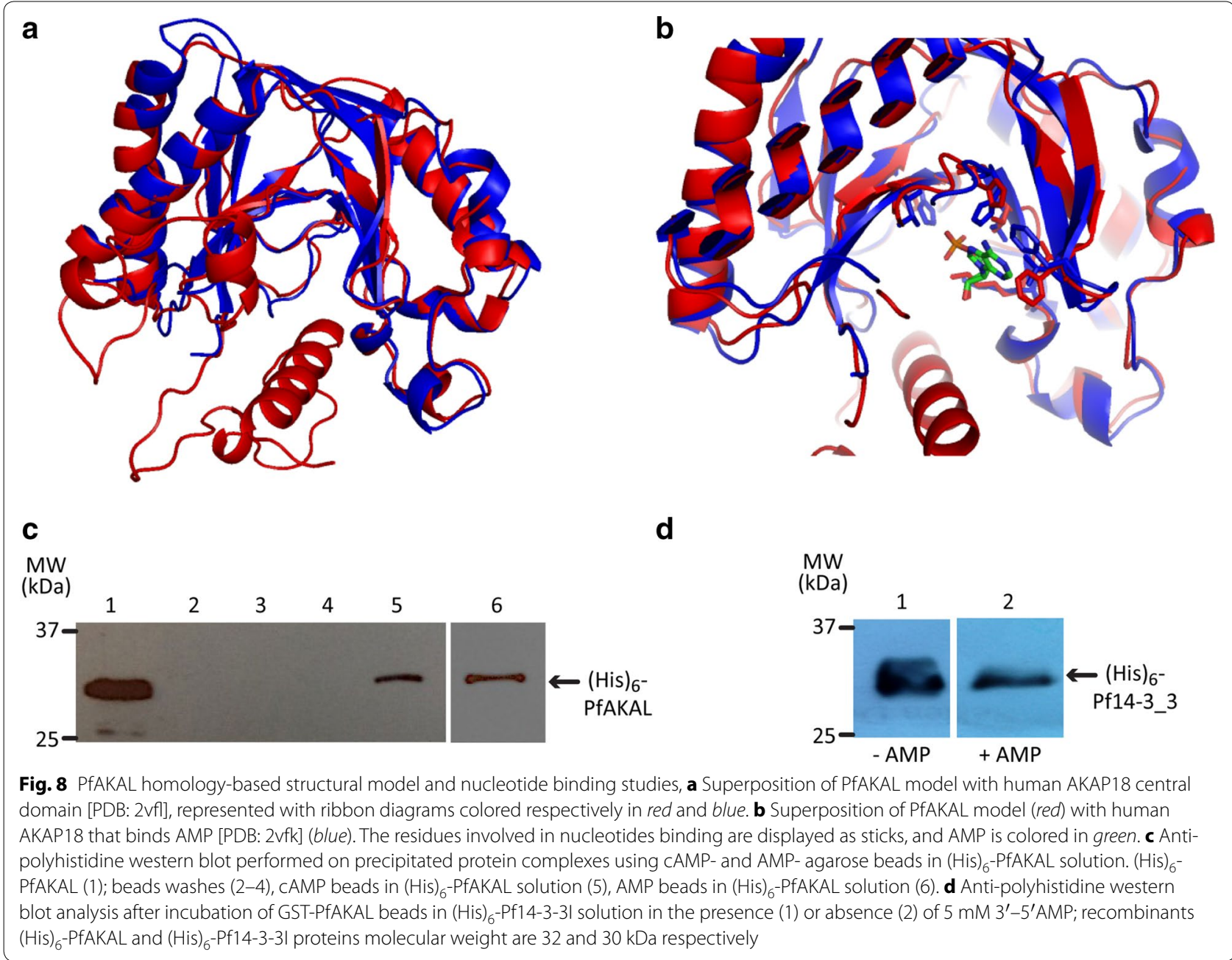

for the protein. One possibility is that PfAKAL acts as a 5'-AMP effector within the parasite, where classically $5^{\prime}$-AMP acts as an energy sensor triggering the AMPactivated protein kinase (AMPK) signalling pathway. In eukaryotic cells, AMPK is ubiquitous and acts as a cellular energy sensor, monitoring the AMP:ATP ratio. If an energy deficit is detected, AMPK activates or inhibits other pathways to restore energy homeostasis [38]. An AMPK has not been identified in P. falciparum until now, but BLAST analysis at PlasmoDB identified a putative actor in the AMPK signalling pathway, PfKIN (PlasmoDB identifier PF3D7_1454300), an as yet uncharacterized protein. Hanson and colleagues have shown that torins, a single structural class of mTOR inhibitors, are potent anti-malarials despite the absence of a recognizable mTOR kinase in Plasmodium. As mTOR kinases are AMPK downstream targets [39], this could suggest the presence of an AMPK signalling pathway in malaria parasites [40]. Thus, a third hypothesis is that PfAKAL acts as an AMP-binder and a scaffolding protein in this putative pathway. Therefore, the interaction of recombinant (His) ${ }_{6}$-PfAKAL with $3^{\prime}-5^{\prime}$ cAMP was investigated (Fig. 8c). But the ability of PfAKAL to hydrolyse cAMP, because of its homology with bacterial phosphoesterases, still need to be established. Different AKAPs such as human AKAP18 $\delta$ and mAKAP, have been shown to interact with PDE [41, 42], and PDE isoforms have been characterized in Plasmodium [11, 12] rendering possible an interaction between PfAKAL and PfPDEs.

\section{Conclusions}

This study presents the characterization of PfAKAL, a protein initially identified as a putative PfPKA anchor. Using molecular and biochemical approaches, we have demonstrated that PfAKAL is expressed in all intraerythrocytic stages, including merozoites and gametocytes, but could not establish any direct protein-protein interaction with PfPKA-R. This suggests an alternative way of anchoring PfPKA within the parasite, and Pf14-3-3I is a PfPKA-R binding partner. The dimerization of Pf14-3-3I 
proteins could stabilize the N-terminus of PfPKA-R promoting the dimerization of PfPKA-R monomers, so creating a docking domain for yet to be identified parasite AKAPs. As Pf14-3-3I is PKA phosphorylated by bovine PKA in vitro, Pf14-3-3I dimerization and/or Pf14-3-3I interaction with PfPKA-R In vivo may be regulated by phosphorylation. The results presented here also highlight structural and biochemical similarities between PfAKAL and human AKAP18y, including nucleotide binding such as AMP and cAMP. PfAKAL may play a role as an AMP sensor, or be involved in other pathways, such as AMPK or PDE pathways.

\begin{abstract}
Abbreviations
AKAP: a-kinase anchoring protein; AKAL: a kinase anchoring protein-like; AMP: adenosine monophosphate; CAMP: cyclic adenosine monophosphate; PKA: protein kinase A; PDE: phosphodiesterase; Ct: cycle treshold; Ni-NTA agarose: nickel-nitrilotriacetic acid agarose; RIPA: radioimmunoprecipitation assay; AMPK: AMP-activated protein kinase.
\end{abstract}

\section{Authors' contributions}

$K B, P B, J-J, L-R, A M$ conceived and designed the experiments. KB, BN, $P B, M L$, $M V, M B, L K, C L, A M$ performed the experiments. $K B, B N, M B, J-J L-R, C L, A M$ analysed the data. KB, BN, PB, MB, J-J, L-R, GL, CL, PD and AM participated in writing the manuscript. The pfakal gene was first identified and cloned in GL's lab, thereafter AM characterized it in PD's lab (UMR216). All authors read and approved the final manuscript.

\section{Author details}

${ }^{1}$ IRD UMR216-MERIT, Faculté de Pharmacie, Mère et enfant face aux Infections Tropicales, Paris, France. ${ }^{2}$ COMUE Sorbonne Paris Cité, Université Paris Descartes, Paris, France. ${ }^{3}$ Laboratoire d'Excellence GR-Ex, Paris, France. ${ }^{4}$ INSERM U1016, Institut Cochin, Paris, France. ${ }^{5}$ CNRS UMR 8104, Faculté de Médecine, Paris, France. ${ }^{6}$ Chimie ParisTech, PSL Research University, Paris, France. ${ }^{7}$ UMR CNRS 8015, Faculté de Pharmacie, Laboratoire de Cristallographie et RMN Biologiques, Paris, France. ${ }^{8}$ CNRS5290, IRD224, University Montpellier 1 \& 2, MIVEGEC, Montpellier, France. ${ }^{9}$ Laboratoire d'Excellence ParaFrap, Paris, France. ${ }^{10}$ Institut Pasteur, Paris, France.

\begin{abstract}
Acknowledgements
We thank Takafumi Tsuboi (Ehime University, Japan) for gift of his wheat germ expression reagents, Jeff Copps and Susan Taylor (UCSD, La Jolla, USA) for peptide array reagents, Susan Taylor (UCSD, USA), Jérôme Clain, Romain Duval, Adrian Luty (UMR216, Paris, France) and Christian Doerig (Monash University, Mebourne, Australia) for helpful discussion and comments, the 3P5 proteomics facility (mass spectrometry profile analyses), and the Animal Housing Facility (In vivo experiments) the Centre de Recherche Pharmaceutique de Paris of the Université Paris Descartes, Sorbonne Paris Cité, the Cellular Imaging facility of the Institut Cochin, Paris (deconvolution microscopy analysis). KB was supported by a Grant from the Région lle de France. This work was supported by the European Commission FP7 (MALSIG project), IRD and Université Paris Descartes.
\end{abstract}

\section{Competing interests}

The authors declare that they have no competing interests.

Received: 3 October 2015 Accepted: 12 April 2016

Published online: 29 April 2016

\section{References}

1. WHO. World Malaria Report. Geneva, World Health Organization; 2015. Available from: http://www.who.int/malaria/publications/ world-malaria-report-2015/report/en/.
2. Enserink M. Malaria's drug miracle in danger. Science. 2010;328:844-6.

3. Yan X, Walkiewicz M, Carlson J, Leiphon L, Grove B. Gravin dynamics regulates the subcellular distribution of PKA. Exp Cell Res. 2009;315:1247-59.

4. Henn V, Edemir B, Stefan E, Wiesner B, Lorenz D, Theilig F, et al. Identification of a novel A-kinase anchoring protein 18 isoform and evidence for its role in the vasopressin-induced aquaporin-2 shuttle in renal principal cells. J Biol Chem. 2004;279:26654-65.

5. Lygren B, Carlson CR, Santamaria K, Lissandron V, McSorley T, Litzenberg J, et al. AKAP complex regulates $\mathrm{Ca}^{2+}$ re-uptake into heart sarcoplasmic reticulum. EMBO Rep. 2007;8:1061-7.

6. Stefan E, Wiesner B, Baillie GS, Mollajew R, Henn V, Lorenz D, et al. Compartmentalization of CAMP-dependent signaling by phosphodiesterase$4 \mathrm{D}$ is involved in the regulation of vasopressin-mediated water reabsorption in renal principal cells. J Am Soc Nephrol. 2007;18:199-212.

7. Gold MG, Smith FD, Scott JD, Barford D. AKAP18 contains a phosphoesterase domain that binds AMP. J Mol Biol. 2008;375:1329-43.

8. Syin C, Parzy D, Traincard F, Boccaccio I, Joshi MB, Lin DT, et al. The H89 CAMP-dependent protein kinase inhibitor blocks Plasmodium falciparum development in infected erythrocytes. Eur J Biochem. 2001;268:4842-9.

9. Merckx A, Nivez M-P, Bouyer G, Alano P, Langsley G, Deitsch K, et al. Plasmodium falciparum regulatory subunit of CAMP-dependent PKA and anion channel conductance. PLOS Pathog. 2008:4:e19.

10. Baker DA. Adenylyl and guanylyl cyclases from the malaria parasite Plasmodium falciparum. IUBMB Life. 2004;56:535-40.

11. Wentzinger L, Bopp S, Tenor H, Klar J, Brun R, Beck HP, et al. Cyclic nucleotide-specific phosphodiesterases of Plasmodium falciparum: pfPDEalpha, a non-essential cGMP-specific PDE that is an integral membrane protein. Int J Parasitol. 2008;38:1625-37.

12. Taylor CJ, McRobert L, Baker DA. Disruption of a Plasmodium falciparum cyclic nucleotide phosphodiesterase gene causes aberrant gametogenesis. Mol Microbiol. 2008;69:110-8.

13. Dastidar EG, Dzeyk K, Krijgsveld J, Malmquist NA, Doerig C, Scherf A, et al. Comprehensive histone phosphorylation analysis and identification of Pf14-3-3 protein as a histone $\mathrm{H} 3$ phosphorylation reader in malaria parasites. PLoS One. 2013;8:e53179.

14. Narum DL, Thomas AW. Differential localization of full-length and processed forms of PF83/AMA-1 an apical membrane antigen of Plasmodium falciparum merozoites. Mol Biochem Parasitol. 1994;67:59-68.

15. Roy A, Kucukural A, Zhang Y. I-TASSER: a unified platform for automated protein structure and function prediction. Nat Protoc. 2010;5:725-38.

16. Zhang Y. I-TASSER server for protein 3D structure prediction. BMC Bioinformatics. 2008;9:40.

17. Brown RL, August SL, Williams CJ, Moss SB. AKAP7gamma is a nuclear RI-binding AKAP. Biochem Biophys Res Commun. 2003;306:394-401.

18. Koonin EV, Gorbalenya AE. Related domains in yeast tRNA ligase, bacteriophage T4 polynucleotide kinase and RNA ligase, and mammalian myelin $2^{\prime}, 3^{\prime}$-cyclic nucleotide phosphohydrolase revealed by amino acid sequence comparison. FEBS Lett. 1990;268:231-4.

19. Walliker D, Quakyi IA, Wellems TE, McCutchan TF, Szarfman A, London WT, et al. Genetic analysis of the human malaria parasite Plasmodium falciparum. Science. 1987;236:1661-6.

20. López-Barragán MJ, Lemieux J, Quiñones M, Williamson KC, Molina-Cruz A, Cui K, et al. Directional gene expression and antisense transcripts in sexual and asexual stages of Plasmodium falciparum. BMC Genom. 2011:12:587.

21. Florens L, Washburn MP, Raine JD, Anthony RM, Grainger M, Haynes JD, et al. A proteomic view of the Plasmodium falciparum life cycle. Nature. 2002;419:520-6.

22. Newlon MG, Roy M, Morikis D, Carr DW, Westphal R, Scott JD, et al. A novel mechanism of PKA anchoring revealed by solution structures of anchoring complexes. EMBO J. 2001;20:1651-62.

23. Gold MG, Lygren B, Dokurno P, Hoshi N, McConnachie G, Taskén K, et al. Molecular basis of AKAP specificity for PKA regulatory subunits. Mol Cell. 2006;24:383-95.

24. McLaughlin WA, Hou T, Taylor SS, Wang W. The identification of novel cyclic AMP-dependent protein kinase anchoring proteins using bioinformatic filters and peptide arrays. Protein Eng Des Sel. 2011;24:333-9.

25. Haste NM, Talabani H, Doo A, Merckx A, Langsley G, Taylor SS. Exploring the Plasmodium falciparum cyclic-adenosine monophosphate (cAMP)dependent protein kinase (PfPKA) as a therapeutic target. Microbes Infect. 2012;14:838-50. 
26. Flaherty BR, Wang Y, Trope EC, Ho TG, Muralidharan V, Kennedy EJ, et al. The stapled AKAP disruptor peptide STAD-2 displays antimalarial activity through a PKA-independent mechanism. PLoS One. 2015;10:e0129239.

27. Peng M, Aye TT, Snel B, van Breukelen B, Scholten A, Heck AJR. Spatial organization in protein kinase a signaling emerged at the base of animal evolution. J Proteome Res. 2015;14:2976-87.

28. Treeck M, Sanders JL, Elias JE, Boothroyd JC. The phosphoproteomes of Plasmodium falciparum and Toxoplasma gondii reveal unusual adaptations within and beyond the parasites' boundaries. Cell Host Microbe. 2011;10:410-9.

29. Lasonder E, Green JL, Camarda G, Talabani H, Holder AA, Langsley G, et al. The Plasmodium falciparum schizont phosphoproteome reveals extensive phosphatidylinositol and CAMP-protein kinase A signaling. J Proteome Res. 2012;11:5323-37.

30. Solyakov L, Halbert J, Alam MM, Semblat J-P, Dorin-Semblat D, Reininge $L$, et al. Global kinomic and phospho-proteomic analyses of the human malaria parasite Plasmodium falciparum. Nat Commun. 2011;2:565.

31. Lam T, Thomas LM, White CA, Li G, Pone EJ, Xu Z, et al. Scaffold functions of 14-3-3 adaptors in B cell immunoglobulin class switch DNA recombination. PLoS One. 2013:8:e80414.

32. Gu Y-M, Jin Y-H, Choi J-K, Baek K-H, Yeo C-Y, Lee K-Y. Protein kinase A phosphorylates and regulates dimerization of 14-3-3 epsilon. FEBS Lett. 2006;580:305-10.

33. Neuberger G, Schneider G, Eisenhaber F. pkaPS: prediction of protein kinase A phosphorylation sites with the simplified kinase-substrate binding model. Biol Direct. 2007;2:1.

34. Moore MJ, Kanter JR, Jones KC, Taylor SS. Phosphorylation of the catalytic subunit of protein kinase A. Autophosphorylation versus phosphorylation by phosphoinositide-dependent kinase-1. J Biol Chem. 2002;277:47878-84.

35. Faul C, Dhume A, Schecter AD, Mundel P. Protein kinase $A, \mathrm{Ca}^{2+} /$ calmodulin-dependent kinase $I I$, and calcineurin regulate the intracellular trafficking of myopodin between the Z-disc and the nucleus of cardiac myocytes. Mol Cell Biol. 2007;27:8215-27.

36. Su C-H, Zhao R, Velazquez-Torres G, Chen J, Gully C, Yeung S-CJ, et al. Nuclear export regulation of COP1 by 14-3-30 in response to DNA damage. Mol Cancer. 2010;9:243.

37. Roy A, Yang J, Zhang Y. COFACTOR: an accurate comparative algorithm for structure-based protein function annotation. Nucleic Acids Res. 2012;40:W471-7.

38. Hardie DG. AMPK-sensing energy while talking to other signaling pathways. Cell Metab. 2014;20:939-52.

39. Kimball SR. Interaction between the AMP-activated protein kinase and mTOR signaling pathways. Med Sci Sports Exerc. 2006;38:1958-64.

40. Hanson KK, Ressurreição AS, Buchholz K, Prudêncio M, Herman-Ornelas JD, Rebelo M, et al. Torins are potent antimalarials that block replenishment of Plasmodium liver stage parasitophorous vacuole membrane proteins. Proc Natl Acad Sci. 2013;110:E2838-47.

41. McSorley T, Stefan E, Henn V, Wiesner B, Baillie GS, Houslay MD, et al. Spatial organisation of AKAP18 and PDE4 isoforms in renal collecting duct principal cells. Eur J Cell Biol. 2006;85:673-8.

42. Rababa'h A, Craft JW Jr, Wijaya CS, Atrooz F, Fan Q, Singh S, et al. Protein kinase $A$ and phosphodiesterase-4D3 binding to coding polymorphisms of cardiac muscle anchoring protein (mAKAP). J Mol Biol. 2013:425:3277-88

\section{Submit your next manuscript to BioMed Central and we will help you at every step:}

- We accept pre-submission inquiries

- Our selector tool helps you to find the most relevant journal

- We provide round the clock customer support

- Convenient online submission

- Thorough peer review

- Inclusion in PubMed and all major indexing services

- Maximum visibility for your research

Submit your manuscript at www.biomedcentral.com/submit 